\title{
Biomechanical and radiographic analysis of a novel, minimally invasive, extension-limiting device for the lumbar spine
}

\author{
Sylvain Palmer, M.D., ${ }^{1}$ Andrew Mahar, M.S., ${ }^{2,3}$ AND Richard OKa, B.A. ${ }^{2}$ \\ ${ }^{\prime}$ Mission Hospital and Regional Medical Center, University of California, Irvine; \\ ${ }^{2}$ Orthopedic Biomechanics Research Center, Children's Hospital, San Diego; \\ and ${ }^{3}$ Department of Orthopedics, University of California, San Diego, California
}

\begin{abstract}
Object. Biomechanical testing and fluoroscopic imaging were used to study an extension-limiting device that has been developed to support and cushion the facet complex. It is a titanium screw-based system with a polycarbonate-urethane bumper that lies against the inferior articular process and is anchored into the pedicle by the screw for posterior dynamic stabilization (PDS).

Methods. Six human cadaveric spines were dissected from L-2 to L-5, leaving all ligamentous structures intact. The intact spines were first tested in flexion and extension, lateral bending, and axial rotation at $\pm 7.5 \mathrm{Nm}$. The PDS devices were inserted at L3-4 and testing was repeated. Fluoroscopic analysis of posterior disc height and foraminal area of the intact and instrumented spines while loaded was performed. All test data were compared using a oneway analysis of variance (statistical significance was set at $\mathrm{p}<0.05$ ).

Instrumented spines had $62 \%$ less motion during flexion and $49 \%$ less motion during extension compared with the intact spines. Neuroimaging analysis showed $84 \%$ less compression of the posterior disc of the instrumented spines during extension, and no difference during flexion compared with intact spines. After instrumentation was affixed, the foraminal area was 36\% larger than in intact spines during extension and $9 \%$ larger during flexion. During axial loading, compression of the posterior disc was decreased by $70 \%$, and analysis showed $10 \%$ decompression prior to loading just from implanting the devices.

Conclusions. The PDS system has the benefit of being a completely percutaneous one, which can be used at all levels of the lumbar spine, including S-1. The PDS system limits spinal motion, enlarges the foramina, and achieves discal decompression.
\end{abstract}

\section{KEY WORDS • spondylosis • posterior dynamic stabilization • instrumentation • minimally invasive surgery $\bullet$ lumbar spine $\bullet$ anatomical study}

$\mathrm{P}$ AINFUL conditions of the aging spine include degenerative disc disease, facet arthropathy, central and foraminal stenosis, and spondylolisthesis. There is significant clinical interest in developing technologies that would alleviate pain while maintaining the spine's natural biomechanics and not resorting to fusion. A novel percutaneous PDS device has been developed by Triage Medical, Inc. that is delivered bilaterally into the superior articular facet of the inferior VB for treating a given spinal motion segment. This device then acts as a mechanical stop between the articulating facets and theoretically should limit extension and thus compression of the central canal, the spinal foramen, and the posterior disc.

This study was designed to evaluate three biomechanical questions concerning this PDS device. First, we wanted to determine the differences in overall stiffness between intact and instrumented spinal motion segments. Second, we also measured the differences in relative inter-

Abbreviations used in this paper: $\mathrm{PDS}=$ posterior dynamic stabilization; $\mathrm{ROM}=$ range of motion; $\mathrm{SD}=$ standard deviation; $\mathrm{VB}=$ vertebral body. vertebral motion between intact and instrumented spinal motion segments. Third, we determined the differences in disc space height and spinal foramen size between intact and instrumented spinal motion segments.

\section{Materials and Methods}

Five male and one female human cadaveric spines, ranging in age from 59 to 85 years (mean age 75 years), were dissected from L-2 to L-5, leaving all ligamentous structures intact. The superior endplate of L-2 and the inferior endplate of L-5 were fixed with a two-part epoxy resin inside custom-designed fixation rigs. Intact spines were first tested in flexion and extension and right and left lateral bending by using a customized cantilevered beam apparatus within a biaxial servohydraulic test frame (model 858; MTS Systems Corp.) (Fig. 1). Loads were delivered from the actuator piston with a sliding cantilevered beam, the functional length of which was given by a linear voltage displacement transducer (SpaceAge Control, Inc.). Angular rotation was calculated from axial displacement of the piston and the changing distance of the specimen attached to the cantilevered beam relative to 


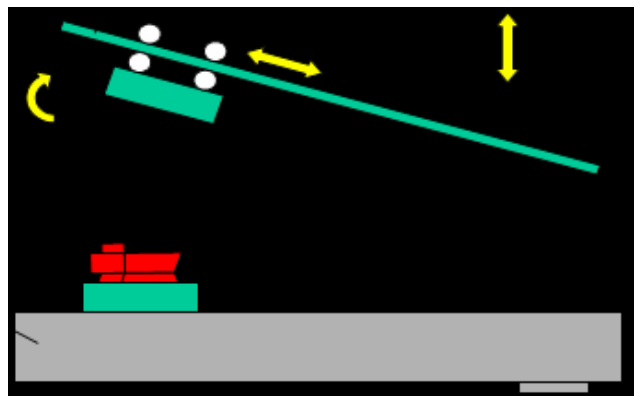

FIG. 1. Schematic depiction of a mechanical testing setup for bending moments. LVDT = linear voltage displacement transducer.

the actuator. A 500-lb tension/compression load-cell (Honeywell Sensotec-Lebow) was mounted on a hinge joint at the end of the piston to measure loads applied to the spine directly. Thus, the applied force and calculated angular rotation provided a real-time calculation of applied torque. A $100-\mathrm{N}$ compressive load and bending moments were applied to $\pm 7.5 \mathrm{Nm}$ for each direction, and each test involved five cycles.

For axial torsion, a 100-N compressive load was also applied to the spine. Each spine was tested between -7.5 and $+7.5 \mathrm{Nm}$ of torsion for five cycles. For all tests, mechanical data for displacement (in millimeters), force (in Newtons), angle (in degrees), and torque (in Newton millimeters) were sampled at $20 \mathrm{~Hz}$ for the duration of each test. The first three cycles served to precondition the specimen, whereas data analysis occurred over the final two cycles. Stiffness (Newton millimeters/degree) was calculated between 0.5 and $7.5 \mathrm{Nm}$ for each spine. Additional data were collected for the intact spines. For noncontact motion measurement, a set of four markers was attached to each VB. The motions of these markers were captured with four $60-\mathrm{Hz}$ infrared cameras and the QTrack motion capture system (Qualisys). Primary rotation and out-of-plane rotations for segments L3-4 (second instrumented motion segment) were reported. After the first stage of mechanical testing, a secondary set of tests was conducted to allow for fluoroscopic analysis. Spines were aligned in a neutral position and then loaded to \pm 7.5 $\mathrm{Nm}$ in flexion and extension. Image-intensified fluoroscopic images were then acquired at each position. Following flexion and extension testing, each spine was then aligned within the test frame for fluoroscopic analysis during axial compression. Image-intensified fluoroscopic images were obtained when each spine was axially loaded at 0 and $700 \mathrm{~N}$. Measurements of posterior disc height (in millimeters) and spinal foramen area (in square millimeters) were calculated from each image (Fig. 2).

After intact testing, the dynamic stabilization devices were implanted using image-intensified fluoroscopy according to the manufacturer's recommendations (Fig. 3). Identical mechanical testing was then repeated for the spines after instrumentation was affixed. Data for overall stiffness, motion between L-3 and L-4, posterior disc height, and spinal foramen area were compared between intact and instrumented spines with a one-way analysis of variance (statistical significance was set at $\mathrm{p}<0.05$ ). Values are given as the mean $\pm \mathrm{SD}$.

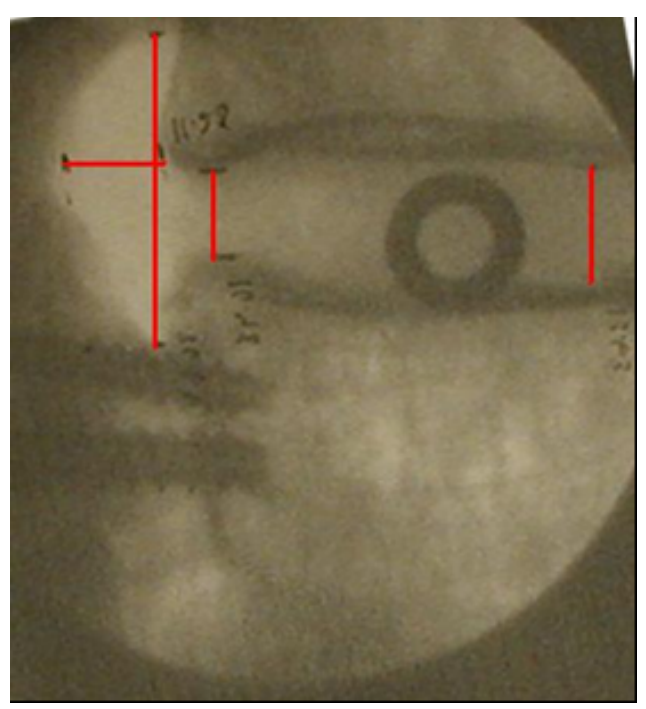

FIG. 2. Fluoroscopic images marked with red lines showing a method of calculating fluoroscopic data.

\section{Results}

\section{Overall Stiffness}

As shown in Table 1, in extension, the instrumented spine (L2-5, $1155 \pm 253 \mathrm{Nmm} /{ }^{\circ}$ ) was slightly less stiff than the intact spine $\left(1363 \pm 143 \mathrm{Nmm} /{ }^{\circ}\right)$. The overall flexion stiffness of the instrumented spine $(681 \pm 84$ $\left.\mathrm{Nmm} /{ }^{\circ}\right)$ was found to be statistically greater $(\mathrm{p}=0.02)$ than for the intact one $\left(486 \pm 101 \mathrm{Nmm} /{ }^{\circ}\right)$. There were no other significant differences reported between the intact and instrumented spines. In left-bending stiffness, the instrumented spine $\left(867 \pm 374 \mathrm{Nmm} /{ }^{\circ}\right)$ was slightly stiffer than the intact one $\left(764 \pm 384 \mathrm{Nmm} /{ }^{\circ}\right)$. This trend was also true for right lateral bending, with the instrumented spine $\left(734 \pm 98 \mathrm{Nmm} /{ }^{\circ}\right)$ slightly stiffer than the intact one $\left(696 \pm 138 \mathrm{Nmm} /{ }^{\circ}\right)$. In axial torsion, there were no differences between left and right directions for each test condition, so these data were pooled prior to analysis. The instrumented spines $\left(761 \pm 111 \mathrm{Nmm} /{ }^{\circ}\right)$ again were slightly stiffer than the intact ones $\left(729 \pm 125 \mathrm{Nmm} /{ }^{\circ}\right)$ in axial torsion.

\section{Relative VB Motion}

Magnitudes of rotation between the L-3 and L-4 VBs were calculated from the neutral position. As shown in Table 2, the instrumented group was found to have a sig-

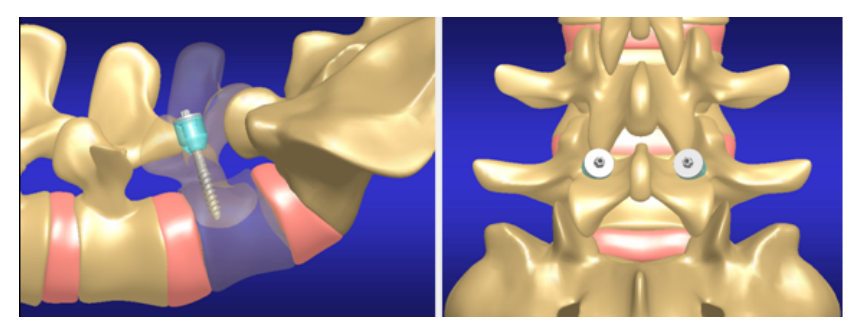

FIG. 3. Three-dimensional reconstructions showing the PDS device in lateral (left) and posteroanterior (right) views. 
TABLE 1

Overall mechanical stiffness $\left(\mathrm{Nmm} /{ }^{\circ}\right)$

for intact and instrumented spines

\begin{tabular}{lccc}
\hline \hline \multirow{2}{*}{\multicolumn{2}{c}{ Motion }} & \multicolumn{2}{c}{ State* $^{*}$} \\
\cline { 2 - 3 } & Intact & Instrumented & p Value \\
\hline flexion & $486.4 \pm 101.2$ & $680.6 \pm 83.6$ & 0.02 \\
extension & $1362.9 \pm 143.3$ & $1155.1 \pm 253.1$ & 0.19 \\
lt bending & $764.1 \pm 383.8$ & $867.1 \pm 374.0$ & 0.68 \\
rt bending & $696.0 \pm 137.8$ & $734.0 \pm 97.7$ & 0.63 \\
axial torsion & $729.0 \pm 125.1$ & $761.3 \pm 110.7$ & 0.55 \\
\hline
\end{tabular}

* Measurements are given as the mean \pm SD.

nificantly lower amount of extension rotation $\left(1.0 \pm 0.3^{\circ}\right)$ compared with the intact state $\left(1.9 \pm 0.8^{\circ}\right)(\mathrm{p}=0.04)$. The instrumented group was also found to have a significantly lower amount of flexion $\left(1.1 \pm 0.3^{\circ}\right)$ compared with the intact group $\left(3.1 \pm 1.2^{\circ}\right)(\mathrm{p}=0.01)$. There were no significant differences for the remaining comparisons. For leftward bending, the instrumented group had a slightly lower ROM $\left(2.3 \pm 0.9^{\circ}\right)$ compared with the intact group $\left(2.7 \pm 1.5^{\circ}\right)$. The overall magnitudes of rightward bending were slightly lower, with the instrumented group (1.6 $\left.\pm 0.5^{\circ}\right)$ again demonstrating slightly lower magnitudes of rotation $\left(2.1 \pm 0.7^{\circ}\right)$. Finally, the reported results for axial torsion very similar between instrumented $\left(4.2 \pm 0.9^{\circ}\right)$ and intact $\left(4.4 \pm 1.1^{\circ}\right)$ groups.

\section{Fluoroscopic Analysis}

Table 3 shows the results of extension and flexion testing. Across all specimens there was a $22 \%$ greater posterior disc height for the instrumented compared with the intact group during extension testing. Also, the foraminal area was 36\% larger during extension for the instrumented group. During flexion testing, the instrumented spines demonstrated minor increases in posterior disc height
TABLE 2

Relative VB motion ( $\left(^{\circ}\right.$ ) between $L-3$ and $L-4$

\begin{tabular}{lccccc}
\hline \hline State* & Flexion & Extension & $\begin{array}{c}\text { Lt } \\
\text { Bending }\end{array}$ & $\begin{array}{c}\text { Rt } \\
\text { Bending }\end{array}$ & $\begin{array}{c}\text { Axial } \\
\text { Torsion }\end{array}$ \\
\hline $\begin{array}{l}\text { intact } \\
\text { mean }\end{array}$ & 3.1 & 1.9 & 2.7 & 2.1 & 4.4 \\
SD & 1.2 & 0.8 & 1.5 & 0.7 & 1.1 \\
$\begin{array}{l}\text { instrumented } \\
\text { mean }\end{array}$ & 1.1 & 1.0 & 2.3 & 1.6 & 4.2 \\
SD & 0.3 & 0.3 & 0.9 & 0.5 & 0.9 \\
p value & 0.01 & 0.04 & 0.61 & 0.27 & 0.79 \\
\hline \multicolumn{7}{l}{ * Measurements are given as the mean \pm SD. }
\end{tabular}

* Measurements are given as the mean $\pm \mathrm{SD}$.

(3\%) and foraminal area (9\%), indicating little influence on normal in vitro biomechanics. Due to the great variability of natural disc heights and foraminal area between specimens, the data recorded for intact and instrumented spines were not statistically different. However, the relative change in each specimen was significantly different between the intact and instrumented states.

To determine the significance between intact and instrumented specimens, the relative change in posterior disc height and foraminal area from the neutral to the extension position was analyzed (Table 4). The change in posterior disc height was measured at $0.64 \mathrm{~mm}$ for the intact state, which was statistically greater $(p=0.002)$ than that of the instrumented state $(0.1 \mathrm{~mm})$. The instrumented spines consistently displayed less motion when moving from a neutral position into extension, and averaged approximately $84 \%$ maintenance of posterior disc height across specimens. The reduction (change) in foraminal area when moving from neutral to extension was also found to be significantly different $(p=0.02)$ between the intact and instrumented states. The change in foraminal area for the intact state $\left(53.2 \mathrm{~mm}^{2}\right)$ was much larger in magnitude than for the instrumented state $\left(23.4 \mathrm{~mm}^{2}\right)$. Overall, there was $56 \%$ less reduction in foraminal area (meaning mainte-

TABLE 3

Fluoroscopic data for extension and flexion testing*

\begin{tabular}{|c|c|c|c|c|c|c|c|c|}
\hline \multirow[b]{2}{*}{ Test } & \multicolumn{4}{|c|}{ Posterior Disc Height (mm) } & \multicolumn{4}{|c|}{ Foraminal Area $\left(\mathrm{mm}^{2}\right)$} \\
\hline & Intact & Instrumented & Diff & $\%$ Diff & Intact & Instrumented & Diff & $\%$ Diff \\
\hline \multicolumn{9}{|c|}{$\begin{array}{l}\text { extension } \\
\text { specimen }\end{array}$} \\
\hline A & 8.34 & 10.18 & 1.84 & 22.06 & 186.73 & 281.29 & 94.56 & 50.64 \\
\hline B & 12.48 & 12.56 & 0.08 & 0.64 & 201.34 & 239.95 & 38.61 & 19.18 \\
\hline $\mathrm{C}$ & 12.72 & 13.66 & 0.94 & 7.39 & 312.52 & 465.63 & 153.11 & 48.99 \\
\hline $\mathrm{D}$ & 3.96 & 5.49 & 1.53 & 38.64 & 297.17 & 340.69 & 43.52 & 14.64 \\
\hline $\mathrm{E}$ & 5.84 & 8.33 & 2.49 & 42.64 & 186.19 & 276.79 & 90.60 & 48.66 \\
\hline \multicolumn{8}{|c|}{$\begin{array}{l}\text { flexion } \\
\text { specimen }\end{array}$} & $36.42 \pm 17.90$ \\
\hline A & 10.76 & 11.3 & 0.54 & 5.02 & 315.48 & 342.97 & 27.49 & 8.71 \\
\hline B & 14.24 & 12.76 & -1.48 & -10.39 & 309.89 & 324.78 & 14.89 & 4.80 \\
\hline $\mathrm{C}$ & 14.12 & 14.01 & -0.11 & -0.78 & 416.73 & 431.07 & 14.34 & 3.44 \\
\hline $\mathrm{D}$ & 5.36 & 6.71 & 1.35 & 25.19 & 347.68 & 361.48 & 13.8 & 3.97 \\
\hline $\mathrm{E}$ & 8.56 & 8.37 & -0.19 & -2.22 & 285.48 & 350.27 & 64.79 & 22.70 \\
\hline $\begin{array}{l}\text { mean } \\
\pm \mathrm{SD}\end{array}$ & $10.61 \pm 3.78$ & $10.63 \pm 3.04$ & $0.02 \pm 1.04$ & $3.36 \pm 13.38$ & $335.05 \pm 50.76$ & $362.11 \pm 40.79$ & $27.06 \pm 21.85$ & $8.72 \pm 8.08$ \\
\hline
\end{tabular}

$*$ Diff $=$ difference 
S. Palmer, A. Mahar, and R. Oka

TABLE 4

Change in posterior disc height and foraminal area from neutral to extension*

\begin{tabular}{|c|c|c|c|c|c|c|c|c|}
\hline \multirow[b]{2}{*}{ Specimen } & \multicolumn{4}{|c|}{ Change in Height (mm) } & \multicolumn{4}{|c|}{ Change in Area (mm) } \\
\hline & Intact & Instrumented & Diff & $\%$ Diff & Intact & Instrumented & Diff & $\%$ Diff \\
\hline A & 0.42 & 0.3 & 0.12 & 28.6 & 70 & 44 & 26 & 37.1 \\
\hline B & 0.4 & 0.04 & 0.36 & 90.0 & 38 & 20 & 18 & 47.4 \\
\hline $\mathrm{C}$ & 0.87 & 0.05 & 0.82 & 94.3 & 36 & 14 & 22 & 61.1 \\
\hline $\mathrm{D}$ & 0.6 & 0.03 & 0.57 & 95.0 & 67 & 3 & 64 & 95.5 \\
\hline $\mathrm{E}$ & 0.91 & 0.08 & 0.83 & 91.2 & 55 & 36 & 19 & 34.5 \\
\hline $\begin{array}{l}\text { mean } \\
\pm \mathrm{SD}\end{array}$ & $0.64 \pm 0.24$ & $0.10 \pm 0.11$ & $0.54 \pm 0.31$ & 84.4 & $53.2 \pm 15.8$ & $23.4 \pm 16.6$ & $29.8 \pm 19.4$ & 56.0 \\
\hline
\end{tabular}

* $. \mathrm{p}=002$ for change of height, and $\mathrm{p}=0.020$ for change of area.

nance of foraminal area) from neutral to extension for the instrumented compared with the intact spines. Viewed conversely, the instrumented spines resulted in a decrease in foraminal area of only $44 \%$ of that seen in the intact specimens during extension.

One interesting comparison in the data is the measurement of posterior disc height at the neutral position for the intact spines and during extension for the instrumented spines. As can be seen in Table 5, there is no significant difference between the two data groups $(p=0.755)$. This demonstrates the ability of the devices not only to decompress the posterior disc initially, but also to maintain the disc height during extension to a degree that, in most cases, is greater than the initial posterior disc height at the neutral position.

For fluoroscopic measurements taken during axial compression, the instrumented group demonstrated an increase in posterior disc height of approximately $10 \%$ when no load $(0 \mathrm{~N})$ was applied (Table 6). Thus, an initial posterior decompression of the motion segment can be achieved simply by implanting the device. When axially loaded to $700 \mathrm{~N}$, the instrumented group had 26\% greater posterior disc height than the intact group, confirming the device's utility as a mechanical block between superior and inferior VBs. Although there were no statistically significant differences found across all specimens due to the relatively high variability of natural disc heights among spines, there is an apparent trend in the data showing the difference between the intact and instrumented groups. However, when measuring the relative change in posterior disc height of each specimen when loaded to $700 \mathrm{~N}$, there were significant differences $(p=0.004)$ found between the intact and instrumented groups. That is, the instrumented spines displayed almost $70 \%$ less posterior disc compression compared with the intact spines when loaded to $700 \mathrm{~N}$.

\section{Discussion}

The degenerating lumbar spine is a source of pain for many individuals. The pain generator can be the disc itself or spondylitic changes in the facet joints. The PDS devices have been designed to address both these mechanisms of pain. There have been two general types of devices: the first is a pedicle-based system such as Dynesys or Isobar; and the second includes interspinous process-based systems such as the DIAM, X STOP, and Wallis. $1,2,4,5,7,8$ Our biomechanical testing has shown that the percutaneous PDS system is most similar to the interspinous process-based systems, although it is based on the facet and pars interarticularis. ${ }^{3}$ The interspinous process spacers have several benefits, but they also have some limitations. Because of anatomical considerations regarding the S-1 spinous process, these implants are not suitable, nor currently recommended, for use at L5-S1. Additionally, even though they are touted as "minimally invasive," all interspinous process spacers currently require a 40 - to 50 -mm incision.

Similar to extension-limiting spacers, Triage Medical's PDS system has been designed to treat the pain caused by degenerative disc disease and spondylosis by stabilizing the lumbar spine without a fusion procedure. Specifically, the PDS system has been designed to prevent extension motion by providing support to the articulating facets. The PDS system is surgically implanted using Triage Medical's proprietary instrumentation. The PDS screws are inserted posteriorly at the base of the superior articular facet, through the pars, and into the pedicles. A countersink is used to remove a small portion of the superior articular facet and the pars interarticularis for the polymeric "bumper" to rest in. The bumpers are advanced, ratcheted over the screws, and wedged between the inferior articular facet of the superior VB and the superior articular facet of the inferior VB. Once the devices are placed bilaterally, the bumpers now act as a stop to prevent extension motion of the facets. The elastic modulus of the material used for the polymeric bumpers is less than that of bone, and it serves to cushion the facets and to relieve stress. Unlike other extension-limiting devices, which require a $40-$ to $50-\mathrm{mm}$ midline incision, the PDS system can be surgically inserted bilaterally through two $15-\mathrm{mm}$ incisions, followed by use of minimally invasive access ports that dilate tissues instead of cutting them. In addition, the PDS system can be used to treat the L5-S1 level, which the interspinous process spacers cannot currently do.

Data from the current study demonstrated similar trends to those reported previously for another dynamic stabilization system, the X STOP. ${ }^{1}$ In that study, an interspinous implant was found to reduce flexion and extension motion by $62 \%$, but there was no effect on lateral bending or axial torsion motion at the instrumented level. The PDS device reduced extension motion by $49 \%$, which was noticeably less than the X STOP, which showed a $62 \%$ reduction in motion during extension. ${ }^{3}$ Unlike the $\mathrm{X}$ STOP, in which the center of rotation for extension motion 
TABLE 5

Posterior disc height ( $\mathrm{mm}$ ) of intact spines in neutral position compared with instrumented spines in extension*

\begin{tabular}{cccc}
\hline \hline Specimen & Neutral (intact) & Extension (instrumented) & $\%$ Diff \\
\hline A & 8.76 & 10.18 & 16.21 \\
B & 12.88 & 12.56 & -2.48 \\
C & 13.59 & 13.66 & 0.52 \\
D & 4.56 & 5.49 & 20.39 \\
E & 6.75 & 8.33 & 23.41 \\
mean \pm SD & $9.31 \pm 3.89$ & $10.04 \pm 3.28$ & $11.61 \pm 11.82$ \\
\hline
\end{tabular}

$* \mathrm{p}=0.755$

is at the spinous process, the center of rotation for the Triage device is at a more natural location, at the base of the facets where the implants are placed. Thus, the Triage devices may allow a more natural ROM while still preventing posterior disc and foraminal compression, thus reducing the probability of stenosis. This may contribute to the smaller degree of reduction in extension ROM for the Triage device compared with the X STOP. Furthermore, the ROM for the specimen as a whole (L2-5) showed little or no difference between instrumented and intact states. This indicates that although the Triage device prevents motion at the instrumented level, it still allows for natural ROM in the specimen as a whole.

The findings in the current study also support the idea that the stenotic segment is placed in slight flexion by the implant, because the insertion of the device resulted in an approximately $10 \%$ increase in posterior disc height when no load was applied to the spine. This initial decompression and the principle of placing the stenotic segment in slight flexion may provide some pain relief associated with simply enlarging the central spinal canal and spinal foramen. Compared with the X STOP, which displayed a $60 \%$ reduction in flexion ROM, the Triage device shows very similar results, with $62 \%$ reduction in flexion ROM. Like the X STOP, lateral bending and axial torsion show little or no difference between intact spines and those treated with the Triage device. Therefore, when the Triage devices were implanted, the specimens were all placed into some degree of flexion (as evidenced by the initial decompression of $\sim 10 \%$ ). For normal spines, putting the specimens in flexion causes tightening of the posterior longitudinal and supraspinous ligaments, resulting in a decrease in ROM during flexion at the instrumented level and an increase in flexion stiffness in the specimen as a whole, which can be seen during testing. In clinical use for spinal stenosis, the devices would be implanted to restore the natural anatomy and kinematics to the treated spine, and probably yield little or no net reduction in flexion motion compared with normal spines.

Pain associated with activities of daily living may also be reduced by limiting potential bulging of the disc or closure of the spinal foramen during extension. ${ }^{6}$ The fluoroscopic data demonstrated that the device maintained a significantly greater posterior disc height compared with the intact state when loaded from neutral to extension. Patients could experience a benefit from the increased foraminal area and maintenance of posterior disc height during flexion and extension motions and during axial compression. During flexion testing, the device appeared to have little effect on posterior disc height $(\sim 3 \%)$. However, during tests in extension, spines treated with the device retained approximately $22 \%$ greater posterior disc height and a nearly $36 \%$ larger area of the spinal foramen. Differences between intact and instrumented states were further confirmed when analyzing data only from neutral into extension positions. Spines in the instrumented state had a significantly lower posterior disc height change ( $84 \%$ less) and much lower magnitude of reduction in foraminal area $(\sim 56 \%$ less), both of which results may yield clinical benefit. During axial compression to $700 \mathrm{~N}$, the posterior disc retained $26 \%$ greater height across all specimens. The relative change in posterior disc height during axial loading was reduced by $70 \%$ in the instrumented spines. This reduction in motion in the posterior disc and foramen may also yield clinical benefits.

\section{Conclusions}

The Triage dynamic stabilization device significantly maintains posterior disc height and foraminal area during extension by limiting the extension motion of the facet joints. The device also stiffens the lumbar spine (L2-5) in flexion and restricts flexion and extension segmental motion without significantly altering motion in lateral bending or axial torsion at the instrumented level. The device performed as intended as a mechanical stop between the articulating facets, limiting extension and therefore limiting compression of the central canal, spinal foramen, and posterior disc. Based on that information,

TABLE 6

Fluoroscopic data from axial compression testing: posterior disc height in $\mathrm{mm}$

\begin{tabular}{|c|c|c|c|c|c|c|c|c|}
\hline \multirow[b]{2}{*}{ Test } & \multicolumn{4}{|c|}{$0 \mathrm{~N}$} & \multicolumn{4}{|c|}{$700 \mathrm{~N}$} \\
\hline & Intact & Instrumented & Diff & $\%$ Diff & Intact & Instrumented & Diff & $\%$ Diff \\
\hline \multicolumn{9}{|c|}{ specimen } \\
\hline A & 5.00 & 5.62 & 0.62 & 12.40 & 4.65 & 5.61 & 0.96 & 20.65 \\
\hline B & 8.01 & 8.15 & 0.14 & 1.75 & 7.26 & 7.95 & 0.69 & 9.50 \\
\hline $\mathrm{C}$ & 8.41 & 8.64 & 0.23 & 2.73 & 7.50 & 8.35 & 0.85 & 11.33 \\
\hline D & 3.88 & 4.47 & 0.59 & 15.21 & 2.94 & 4.06 & 1.12 & 38.10 \\
\hline E & 4.40 & 5.10 & 0.70 & 15.91 & 3.26 & 4.65 & 1.39 & 42.64 \\
\hline $\mathrm{F}$ & 1.74 & 1.92 & 0.18 & 10.34 & 1.23 & 1.67 & 0.44 & 35.77 \\
\hline $\begin{array}{c}\text { mean } \\
\pm \mathrm{SD}\end{array}$ & $5.24 \pm 2.55$ & $5.65 \pm 2.48$ & $0.41 \pm 0.25$ & $9.72 \pm 6.14$ & $4.47 \pm 2.50$ & $5.38 \pm 2.51$ & $0.91 \pm 0.33$ & $26.33 \pm 14.38$ \\
\hline
\end{tabular}


we infer that this device would potentially alleviate symptoms associated with spinal stenosis and discogenic back pain.

\section{Disclosure}

Dr. Palmer has a financial interest in Triage Medical, whereas Andrew Maher and Richard Oka do not.

\section{References}

1. Christie S, Song J, Fessler R: Dynamic interspinous process technology. Spine 30 (16 Suppl): S73-S78, 2005

2. Lee J, Hida K, Seki T, Iwasaki Y, Minoru A: An interspinous process distractor (X STOP) for lumbar spinal stenosis in elderly patients: preliminary experiences in 10 consecutive cases. J Spinal Disord Tech 17:72-77, 2004

3. Lindsey DP, Swanson KE, Fuchs P, Hsu KY, Zucherman JF, Yerby SA: The effects of an interspinous implant on the kinematics of the instrumented and adjacent levels in the lumbar spine. Spine 28:2192-2197, 2003

4. Schiavone AM, Pasquale G: The use of disc assistance prostheses (Diam) in degenerative lumbar pathology: indications, technique, and results. Ital J Spinal Disord 3:213-220, 2003

5. Sénégas J: Mechanical supplementation by non-rigid fixation in degenerative intervertebral lumbar segments: the Wallis system. Eur Spine J 11 (2 Suppl): 164-169, 2002

6. Swanson KE, Lindsey DP, Hsu KY, Zuckerman JF, Yerby SA: The effect of interspinous process implant on intervertebral disc pressure. Spine 28:26-32, 2003

7. Zucherman JF, Hsu KY, Hartjen CA, Mehalic TF, Implicito DA, Martin MJ, et al: A multicenter, prospective randomized trial evaluating the $\mathrm{X}$ Stop interspinous process decompression system for the treatment of neurogenic intermittent claudication: two-year follow-up results. Spine 30:1351-1358, 2005

8. Zucherman JF, Hsu K, Hartjen CA, Mehalic TF, Implicito DA, Martin MJ, et al: A prospective multi-center randomized study for the treatment of lumbar canal stenosis with the X STOP interspinous implant: 1-year results. Eur Spine J 13:22-31, 2004

Manuscript submitted October 5, 2006.

Accepted November 22, 2006.

This study was funded by the Triage Medical Corporation, Irvine, California.

Address reprint requests to: Sylvain Palmer, M.D., 26732 Crown Valley Parkway, Suite 561, Mission Viejo, California. email: sylvainpalmer@cox.net. 